Original Research Paper

\title{
Physiologic Human Fluids and Swelling Behavior of Hydrophilic Biocompatible Hybrid Ceramo-Polymeric Materials
}

\author{
${ }^{1}$ Aversa Raffaella, ${ }^{2}$ Relly Victoria V. Petrescu, ${ }^{1}$ Apicella Antonio and ${ }^{2}$ Florian Ion T. Petrescu \\ ${ }^{1}$ Advanced Material Lab, Department of Architecture and Industrial Design, \\ Second University of Naples, 81031 Aversa (CE) Italy \\ ${ }^{2}$ Bucharest Polytechnic University, Bucharest, Romania
}

Article history

Received: 15-10-2016

Revised: 04-11-2016

Accepted: 05-11-2016

Corresponding Author: Apicella Antonio

Advanced Material Lab, Department of Architecture and Industrial Design, Second University of Naples, 81031

Aversa (CE) Italy

Email: antonio.apicella@unina2.it

\begin{abstract}
All synthetic and natural materials to be used in biomedical applications that involve the contact with human body need to be investigated for their physical and chemical modification induced by the human physiological fluids contact and sorption. The development and testing in human physiological equivalent fluids of new hybrid biomaterials are presented. The role of water and its equilibrium modification in the human physiology is discussed and the swelling and sorption behavior in the physiological environment of a nanostructured and osteoconductive biomaterials based on Poly-Hydroxyl-Ethyl-Meth Acrylate matrix (pHEMA) filled with fumed amorphous nanosilica particles is presented. This material differently swells in presence of aqueous physiological solution fluid. Biological hybrid scaffolds for bone regeneration and growth made using synthetic materials able to correctly interact with the physiological fluids while inducing the growth of biological tissues may favor the birth in the medical field of a new class of hybrid materials. Our multidisciplinary approach explores in the this paper, novel ideas in modeling, design and fabrication of new nanostructured scaffolding biomaterials with enhanced functionality and improved interaction with $\mathrm{OB}$ cells.
\end{abstract}

Keywords: Biomaterials, Bioactive Scaffolds, Nanocomposites, Tissue Engineering

\section{Introduction}

Innovative tissue engineering biomimetic materials based on hydrophilic polymers have been reported to show attractive physical, biological and mechanical properties for several biomedical applications (Montheard et al., 1992; Filmon et al., 2002; Davis et al., 1991; Kabra et al., 1991; Apicella et al., 1993; Peluso et al., 1997).

Aqueous solutions containing salts and organics represent the principal component of the human physiological fluids. All synthetic and natural hydrophilic materials used in biomedical application should be investigated and characterized for their interactions with aqueous environments. Water origin, function and how it is influenced by organic and clinical pathologies need to be understood for a correct utilization of the biomaterials.

Humans adults are made of water, almost $65 \%$ of everything we stand for as human beings is water, but this content is highly variable during its life span; born rich of water that then gradually is lost as getting older. A human embryo is $97 \%$ water, at birth the newborn is made up of $90 \%$ water, toddler contains $80 \%$ water, the same child at school age will have $70 \%$ water, following that in adulthood to remain only $60-65 \%$ water by weight.

Different amounts of water are present in the composition of all organs to help them to perform their functions. Blood contains 90\% water, muscles 75\%, 83\% brain, lungs $86 \%$, 83\% kidneys, eyes $95 \%, 22 \%$ bones. Kidneys need water in order to filter the blood, digestive needs water for digestion, skin need water to keep smooth and healthy and bones to maintain elasticity and strength (Babaev et al., 2010).

Moreover, the percentage of water differs also for sex and body fat percentage. Females have a higher percentage of fat (retained in the hands, hips and the subcutaneous fat cellular tissue) than men; they will average $5 \%$ less water than men of the same age. This is because fat tissue has the ability to retain a very small 
amount of water, which explains the reduction of the body's water content.

Water is the medium that holds all biological and chemical reactions in the body, resulting in the production of heat and energy metabolism reactions that are required for life support. In order to allow these reactions occurring it is necessary that several organic and inorganic substances should dissolve in the aqueous medium reducing the water molecules activity.

Moreover, all these dissolved substances, which are necessary for the human metabolism, may also interact with the hydrophilic biomaterials that have been introduced in the human body.

Bio-functional and mechanical properties of the prosthetic materials can be then altered or, conversely, they can alter the physiological equilibrium altering the aqueous medium composition.

Water circulates through the blood and lymph oxygen and nutrients to the organs. Water helps remove metabolic waste by the kidneys, which is impossible in the absence of a quantity of water acting as a conductor. Water participates in the formation and the growth of cells of the body. The water helps in maintaining a constant body temperature by making the body thermoregulation. When the body is overheated (summer or in fever) water removes heat which abound through perspiration and in winter when the body suffers of cold, water adapts the body to keep warm blood around vital organs, causing capillaries to contract to save water losses (Buzea et al., 2015; Petrescu et al., 2015).

When water is present at the cellular level in small quantity, the cells (mitochondria) cannot produce enough energy. Once the numbers of energy molecules (ATP) are decreased, the body is getting weaker, aging and death. Water loss triggers thirst reflex (that means it has already begun the process of dehydration and body suffer). Sometimes that thirst not works (to be inhibited by factors such as age, hormonal disorders, or a chaotic lifestyle, but that does not mean that the body does not require hydration). In addition dehydrated and tired brain refuses to send appropriate signals for thirst. Therefore it requires some water consumption calculated from time to time whether we are thirsty or not.

The loss from and water content modification in of the cells (cellular water loss) with age is a serious problem for several organs and it can alter the functional behavior of biomedical apparati.

Water lost can seriously affect the natural functionalities of the brain and of the nervous system, leading to the appearance of symptoms like nervousness, irritability and anxiety, tiredness, exhaustion and inability to concentrate.

Because a dehydrated brain cannot correctly process information then intellectual performances are reduced, i.e., short-term memory could be impaired for a decrease of only $2 \%$ of body water. When the hydration level of the body decreases, lungs slow down their activity thus limiting water loss through breathing. Appear difficulty in breathing and organs are not functioning properly oxygenated in damage (dizziness and general bad).

It can be inferred that the water content in the human body may be subjected to significant variations that depends of diverse and uncontrolled factors.

Materials used in biomedical applications such as prostheses and scaffolds for tissue engineering may be positively or adversely affected by the presence and fluctuation of extracellular body water content.

Aversa et al. (2016a) developed highly biocompatible new hybrid materials that are based on the use of fumed amorphous silica nanoparticles and a hydrophilic poly(hydroxyl-ethyl-methacrylate) (pHEMA) that can be used for bone scaffolding materials. The addition of fumed silica improved the self-organization of the polymeric network since it increase the number of hydrogen bonds between the polymer hydroxyl functional groups with the oxigens of the silica nanoparticles. Due to these internal bonding, the resulting nano-filled composites increases its stiffness while remains transparent showing final improved mechanical strength (Schiraldi et al., 2004). This occurrence (the surprisingly high increase of the material stiffness) overcomes one of the major problems in the application of hydrogels that are associated with their low mechanical characteristics. Early studies confirmed that the these hybrid nano-composites acquire the biomimetic and osteoconductive properties that are needed to design mechanically bioactive scaffolding systems for Osteoblast growth (Schiraldi et al., 2004). In healthy conditions, modeling and remodeling collaborate to obtain a correct shape and function of bones. In a bone implanted with a rigid metallic prosthesis, even if functionality is restored, the biomechanical equilibrium and stress distribution are significantly altered (Aversa et al., 2009; Sorrentino et al., 2007; 2009). Loads on bones cause bone deformations that generate signals that some $\mathrm{OB}$ cells can sense and respond to. Bone modeling and remodeling are driven and controlled by these threshold signals (Wolff, 1892; Frost, $1964 ; 1990 ; 1994 ; 2004)$. Remodeling processes repair the injury by removing and replacing the damaged tissues with new bone. Moreover, excessive or insufficient loading alters such remodeling process (Frost, 1994). Early studies by Wolff (1892) stated that the bone mechanical response could induce modifications of its architecture. Frost (1964) found mathematical relationships describing the modification of the bone tissue under specific loading that quantitatively accounted for bone deformations (Frost, 2004). Remodeling processes repair the bone by replacing the damaged tissues with new structured bone.

The use of biocompatible and biomechanically active material that can be "designed" to reproduce bone compatible and biomimetic structural properties in 
mutating physiological conditions is discussed in the present paper.

\section{Materials and Methods}

\section{Materials}

A commercial 2-hydroxyethyl methacrylate (HEMA), obtained from Sigma-Aldrich Chemicals Co., (St. Louis, MO, USA) has been used as hydrophilic matrix. Fumed silicon dioxide (Aerosil 300 Degussa, Germany) with a mean diameter of $7 \mathrm{~nm}$ and specific surface area of $300 \mathrm{~m}^{2} \cdot \mathrm{g}^{-1}$ was utilized as bioactive filler. The $\alpha-\alpha$ ' azoisobutyrronitrile (AIBN), obtained from Fluka (Milan, Italy) has been utilised as initiator of the radical polymerization reaction. HEMA monomers were mixed with the fumed silica in the ratio of 5, 10, 15, 20 and $25 \%$ by volume. The resin was degassed and transferred in $2.5 \mathrm{~mm}$ thick planar moulds and then polymerized in temperature controlled oven set at $60^{\circ} \mathrm{C}$ for $24 \mathrm{~h}$ with a final post-cure of $1 \mathrm{~h}$ at $90^{\circ} \mathrm{C}$.

\section{Biomimetic/Biomechanical Approach}

Our Biomimetic and Biomechanical approach resulted from a parallel physiological, mechanical and physical characterization of the hydrophilic hybrid material in presence of aqueous environment simulating physiological fluids.

Physiologically, about $2 / 3$ of the total water in a human body is located in the intracellular compartment (water that is kept as a reserve within any human cell). The extracellular water is one third and it is so distributed: $20 \%$ extra vascular sector (Lymph and interstitial fluid) and $8 \%$ vascular sector (the water from blood). Water molecules diffuse freely and quickly between all these compartments in response to changes of the solvents concentrations, to maintaining the balance between compartments (osmosis). Similar, osmotic and diffusive phenomena have been described to occur in polymeric materials (Holley et al., 1970; Aversa and Apicella, 2016).

In an adult of $70 \mathrm{~kg}$, water is of about $40 \mathrm{l}$, of which 251 is intracellular water, extracellular water $15 \mathrm{l}$; in the total plasma volume is typically 2.75 to 31 . The water intravascular is at least $85 \%$ in the region of the capillary and venous and arterial within $15 \%$. Of maintaining the volume of arterial water depends greatly the arterial blood pressure, regulation of extracellular fluid volume and retention of sodium and water.

Sodium is the main extracellular cation and the determining element of achieving an effective osmosis. The intracellular sodium concentration is an average of $15 \mathrm{mmol} / \mathrm{l}$ and the extracellular sodium $140 \mathrm{mmol} / \mathrm{l}$. The average daily intake of sodium is 100-200 mmol. Sodium absorption occurs in the small intestine and colon. Body water (intra and extracellular) comes on one side of liquids and foods (1-4 1/day) and on the other side of the oxidation of lipids, carbohydrates and proteins (200-500 g water daily).

Water and dissolved ions sorption can strongly influence the osmotic tension generated in a polymeric materials (Aversa and Apicella, 2016). Two limiting like liquid based on $\mathrm{NaCl}$ solution comprising of isotonic $(0.15 \mathrm{M})$ and hypotonic solutions $(0.5 \mathrm{M})$ have been used in our study to simulate extracellular physiological fluid behaviour.

The hydrophilic hybrid material prepared in our study strongly interacts with physiological aqueous solutions. Figure 1 shows the physical phenomena occurring in a 10 $\mathrm{mm}$ long and $10 \mathrm{~mm}$ diameter cylinder of our materials once exposed to an aqueous medium (distilled water).

The initially glassy sample when immersed in plain distilled water (water activity is unity) progressively swells, due to water molecules diffusion and sorption, finally reaching an equilibrium swollen and rubbery state (right lower hand in Fig. 1). The process is characterized by the presence of a clear front that separates an unaffected and water unpenetrated glassy core and a surrounding swollen and water molecules penetrated outer shell (left lower hand in Fig. 1).

This particular sorption behaviour has been deeply investigated in the past (Holley et al., 1970; Apicella and Hopfenberg, 1982) and it is referred as Case II sorption (where a state change occurs) to differentiate it from the classical and ordinary Fickian sorption (where the diffusion of the penetrant does not induce any physical modifications).

Upon immersion in the aqueous medium, this anomalous sorption steadily proceeds up to the final complete swelling of the sample; as the immersion time is prolonged, in fact, the unaffected glassy core reduces its thickness (upper part of Fig. 1).

\section{Mechanical Characterization}

Shear elastic moduli, which were measured on dry and swollen in different concentration $\mathrm{NaCl}$ solutions, of p-HEMA Hybrid nano-composites were performed using a METTLER-TOLEDO (Zurich, Switzerland) dynamical mechanical tester (DMA) operating in shear mode. The elastic and viscous components of the shear modulus were measured under constant frequency in isothermal condition. The dry samples were dried under vacuum at a $60^{\circ} \mathrm{C}$ for $24 \mathrm{~h}$ before testing. In the shear test mode, the $10 \mathrm{~mm}$ diameter and 2 sample disks, which were cut from the polymeric slab, were placed between three steel plates forming a symmetrical sandwich. Isothermal scans at $37^{\circ} \mathrm{C}$ in a dry Nitrogen purged environment were performed. The deformation control was set to $10 \mu \mathrm{m}$ and a force limitation of $0,9 \mathrm{~N}$ was applied at an oscillating frequency of $10 \mathrm{~Hz}$.

The same procedure was applied for the swollen polymer (without drying stage and with force limitation of $0.09 \mathrm{~N}$ ). 


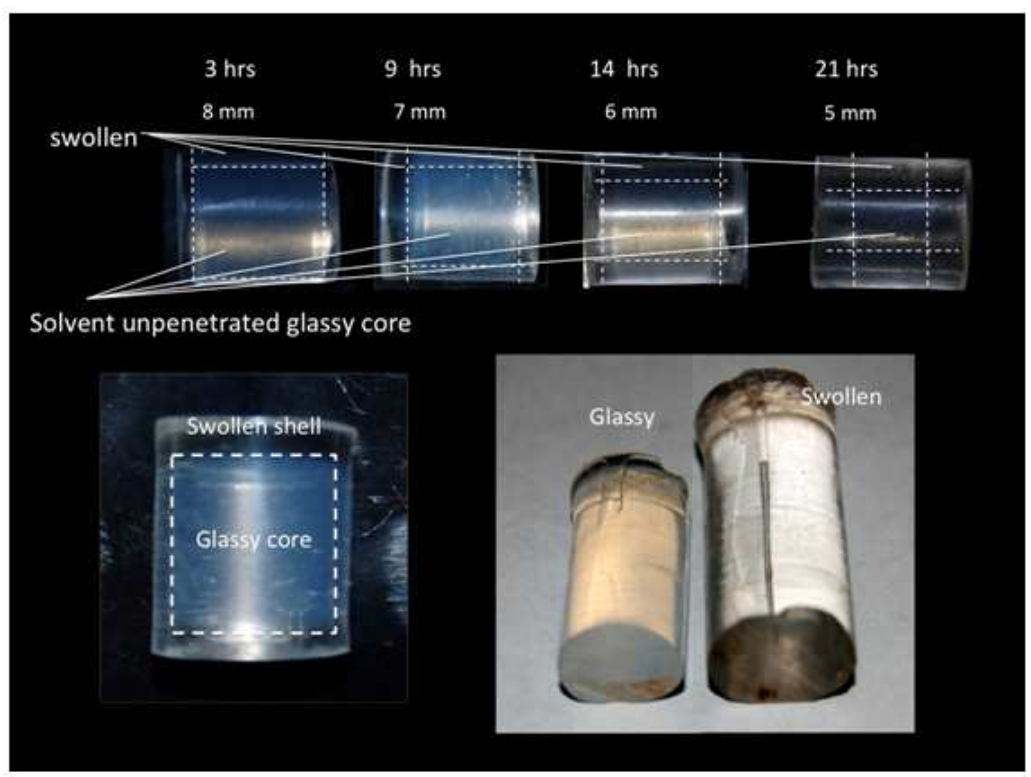

Fig. 1. Swelling behavior of our hybrid hydrophilic nanocomposite in water

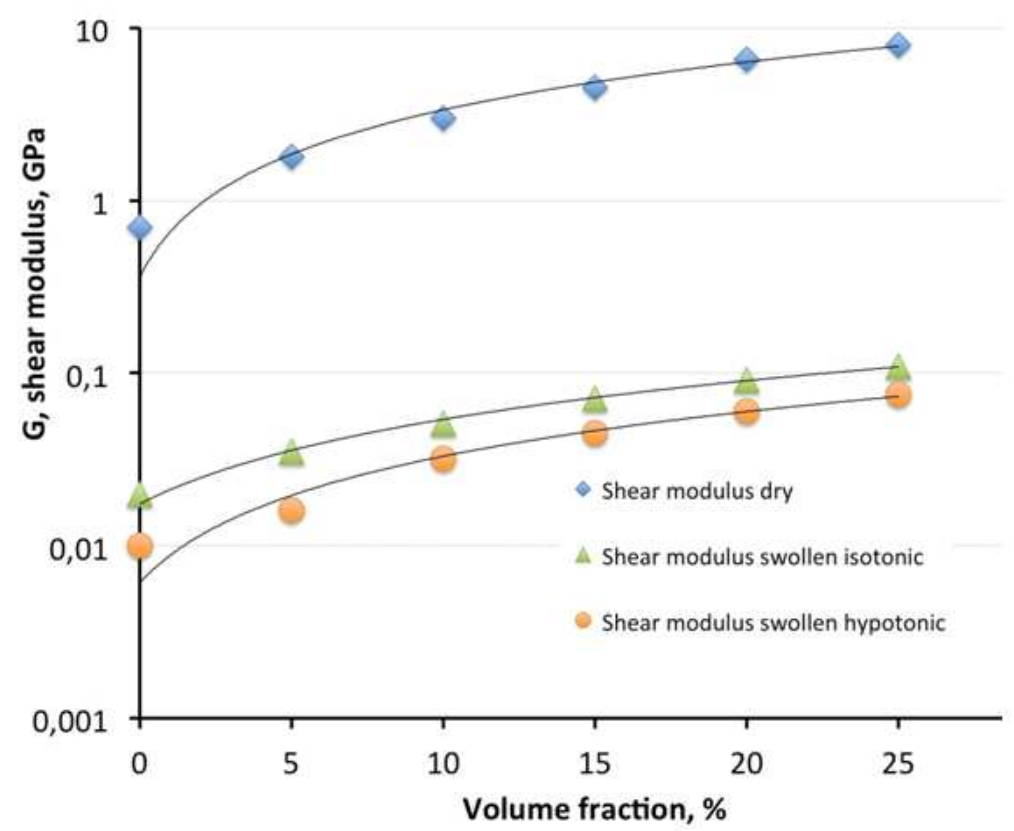

Fig. 2. Shear moduli of dry and swollen (rubbery) of our hybrid hydrophilic nanocomposites equilibrated in hypotonic and isotonic aqueous solutions

The dry and saturated in hypotonic and isotonic Hybrid pHEMA nanocomposites with compositions ranging from 5 to $25 \%$ by volume of nanosilica were isothermally shear tested in a Dynamic Mechanical Analyser operating under a Nitrogen dry atmosphere at $10 \mathrm{~Hz}$ and $37^{\circ} \mathrm{C}$.

The shear moduli of the dry and swollen samples for the samples of different compositions are plotted in Fig. 2 in a logarithmic scale.
A significant plasticization effect was induced by the equilibration in the saline physiological solutions. The Shear moduli of the dry and glassy samples range from 0.8 to $9 \mathrm{GPa}$ while they range from 0.01 to $0.1 \mathrm{GPa}$ for the softer and rubbery swollen samples. The less concentrated hypotonic aqueous solution induced a higher level of plasticization (lower moduli).

Moreover, the samples showed a predominantly elastic behavior (the viscous component was negligible 
for all compositions). It has been demonstrated by Aversa and Aversa (2016) that the measured shear moduli of pHEMA-Nanosilica composites of different composition do not follow classical Halpin and Kardos (1976) equation for particulate composites. A linear dependency of shear modulus values at progressively increasing content of nanosilica loading was described, instead. This unexpected behaviour confirmed the hybrid nature of our nanosilica pHEMA composites.

The mechanical shear moduli of the dry and swollen samples reported in Fig. 2 have been then interpolated by linear fitting (full lines in Fig. 2).

To reach the mechanical properties requirements needed for bone replacement, the nanofiller/p-HEMA compositions for suitable hybrid nanocomposites are:

- When glassy and dry it should be comparable, during the surgical stages, to the rigidity of the bone $(G=2$ $4 \mathrm{GPa})$

- Once swollen it should be comparable, during osteointegration stage, to cartilage and ligament elasticity $10-40 \mathrm{MPa}$

Shear moduli comparable to those of the cortical bone have been measured in the dry state for nanosilica volumetric fractions ranging from 5 and $10 \%$. A volume fraction of $5 \%$ has been then chosen for sample preparation and sorption behaviour characterization.

Shear moduli of 16 and $35 \mathrm{MPa}$ have been experimentally found for the hybrid composite $(5 \%$ by volume) swollen in isotonic and hypotonic solutions, respectively. This value turns comparable to that of the periodontal ligament strained in the same conditions and to that of an articular cartilage (Toyrasa et al., 2001; Comerun, 1986).

\section{Sorption and Swelling Tests}

Planar $2.0 \mathrm{~mm}$ thick and $20 \times 20 \mathrm{~mm}$ samples of the hybrid nanocomposite with $5 \%$ of nanosilica, which have been prepared according to the conditions illustrated in the Material section, were used for the characterization of the anomalous sorption kinetics and equilibria in aqueous physiological isotonic and ipotonic saline $(0.5$ and $0.15 \mathrm{M} \mathrm{NaCl})$ solutions. These two solutions were chosen to simulate two possible different physiological extracellular fluid conditions that may be encountered for people age, sex or physical differences.

As previously discussed, in fact, the percentage of water in extracellular fluid differs for sex and body fat percentage. Females, which have a higher percentage of fat than men, average $5 \%$ less water than men of the same age.

The aqueous solution uptakes in the initially dry samples were determined at equilibrium by gravimetric measurements in a $0.1 \mathrm{mg}$ precision Mettler Toledo analytical balance and are reported in Fig. 3 .
Analogously, the advancement and penetration of the aqueous medium swelling fronts in the limiting Case II anomalous sorption (Nicolais et al., 1984; Apicella and Hopfenberg, 1982) observed in our hydrophilic samples were monitored as a function of time by measuring, with a Leitz stereo-microscope, the thickness of the unswollen residual glassy core (Fig. 4) and the overall thickness \% increases along the $\mathrm{Z}$ axis and \% lengths variations along the two orthogonal axes (X and $Y$ ) in the plan of the sample slab (Fig. 6).

All the equilibrium sorption and swelling experiments were performed at $37^{\circ} \mathrm{C}$ (thermostatic water bath) until constant weight up-take was reached (100 h).

The sorption behaviour in a physiological isotonic and hypotonic solutions of our $5 \%$ volume fraction glassy hybrid materials has been investigated for both weight uptakes and swelling kinetics. Sorption and swelling kinetics were plotted as a function of time (Fig. 3,4 and 6) and of the square root of time (Fig. 5).

Fig. 3 shows the raw sorption weight uptakes data in function of the immersion time in the two hypotonic and isotonic physiological solutions.

The 5\% hybrid nanocomposite dramatically swells in isotonic water solution (Fig. 3) picking up $42-45 \%$ of its dry weight and reducing its shear modulus to $15-25 \mathrm{MPa}$ (as measured in DMA tests of Fig. 2). Such phenomenon is associated to the water induced polymer plasticization that reduces the polymer glass transition temperature below the test temperature.

Once exposed to the aqueous solution, the initially dry and glassy pHEMA nanocomposite $2 \mathrm{~mm}$ thick plates starts to swell showing a clear front dividing the rubber-swollen outer portion and the unaffected glassy core. The glassy core thickness progressively reduces as the swollen front advance through the sample. A measure of the swelling kinetic, which has been reported in Fig. 4 as a function of the time and in Fig. 5 as a function of the square root of time, is given by the rate of reduction of the glassy core thickness. The observed swelling front initially advanced at constant rate according to the limiting relaxation controlled anomalous sorption mechanism indicated as "Case II sorption" (Nicolais et al., 1984).

The initial linear swelling rate (Fig. 4) is slower for the isotonic solution, about $0.10 \mathrm{~mm} / \mathrm{h}$ and faster, about $0.13 \mathrm{~mm} / \mathrm{h}$, for the hypotonic solution sorption. As swelling proceeds further on, however, diffusive resistance for water molecules flow develops in the outer swollen layer reducing the swelling rate of the remaining glassy core (Apicella and Hopfenberg, 1982).

Once the glassy core disappears (indicated by an arrow in Fig. 5), namely after 425 and $500 \mathrm{~min}$ (see right hand side of Fig. 5) for the hypotonic and isotonic solution respectively, sample weight uptakes are about $25 \%$ for both 
samples and they continues to steadily increase up to final equilibrium values that are 45 and $42 \%$ (left hand side of Fig. 5) for the less concentrated hypotonic and more concentrated isotonic solutions, respectively.

This occurrence is related to the achievement of a complete swelling equilibrium once the glassy core is not more constraining the deformations along the $\mathrm{X}$ and Y axes of the sample slab (Fig. 6).

This peculiar behaviour can be inferred from the swelling data reported in Fig. 6 where the swelling percentage in the $\mathrm{X}, \mathrm{Y}$ and $\mathrm{Z}$ directions (right hand side of Fig. 6) are reported and compared with the core thickness reduction (left hand side of Fig. 6).
Along the Z-axis (through the thickness), the swelling curve reaches a first plateau of $12 \%$ in correspondence of the glassy core exhaustions and then reprises to increase.

Along the $\mathrm{X}$ and $\mathrm{Y}$-axes, the swelling is initially inhibited by the presence of the stiff glassy core. Once disappeared, however, the swelling starts and reach its final value of about $12 \%$. The swelling along the thickness, conversely, continues after the meeting of the swelling fronts and reach a final value of $18 \%$.

This evidence indicates that a material plastic deformation occurred in the $\mathrm{Z}$ direction.

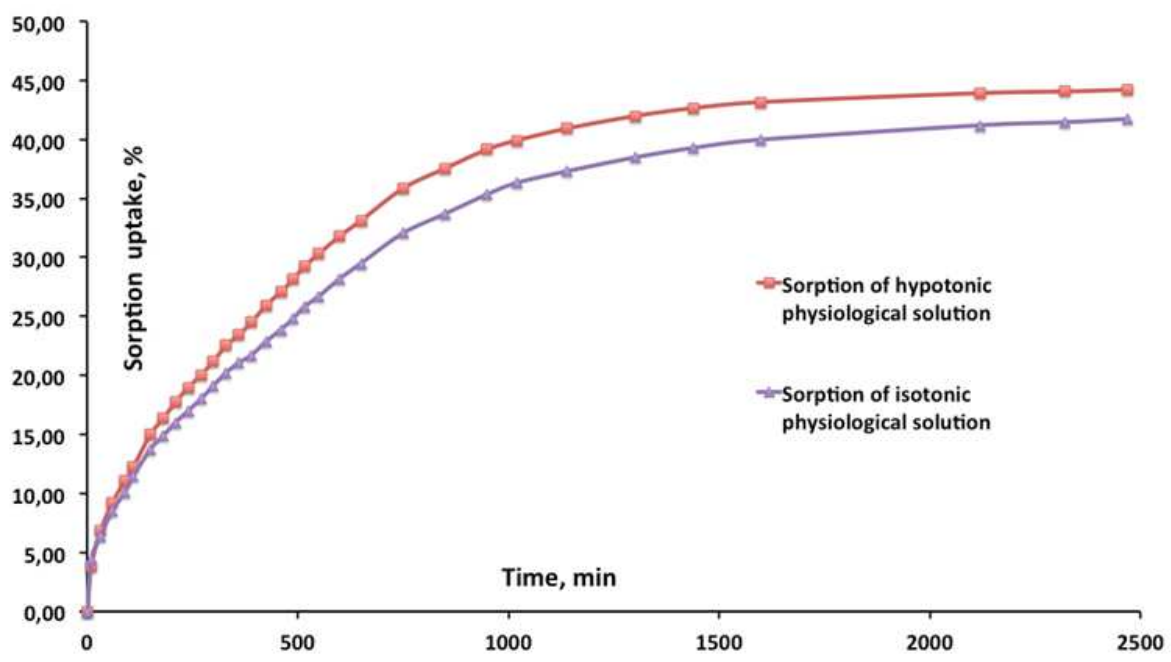

Fig. 3. Swelling and sorption kinetic in a $5 \%$ by volume hybrid nanocomposite in physiological $0.05 \mathrm{M}$ (hypotonic) and $0.15 \mathrm{M}$ (isotonic) $\mathrm{NaCl}$ solutions

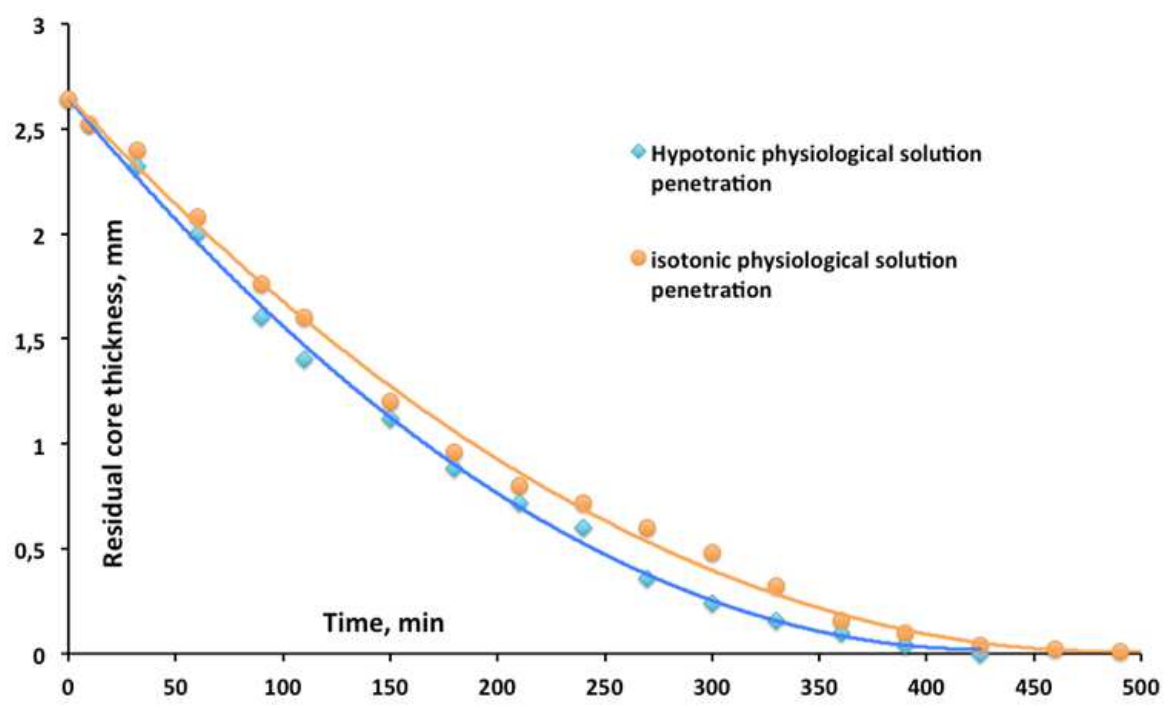

Fig. 4. Residual glassy core thickness reduction kinetic in a $5 \%$ by volume hybrid nanocomposite swelled in physiological $0.05 \mathrm{M}$ (blue circle, hypotonic) and $0.15 \mathrm{M}$ (orange circle, isotonic) $\mathrm{NaCl}$ solutions 


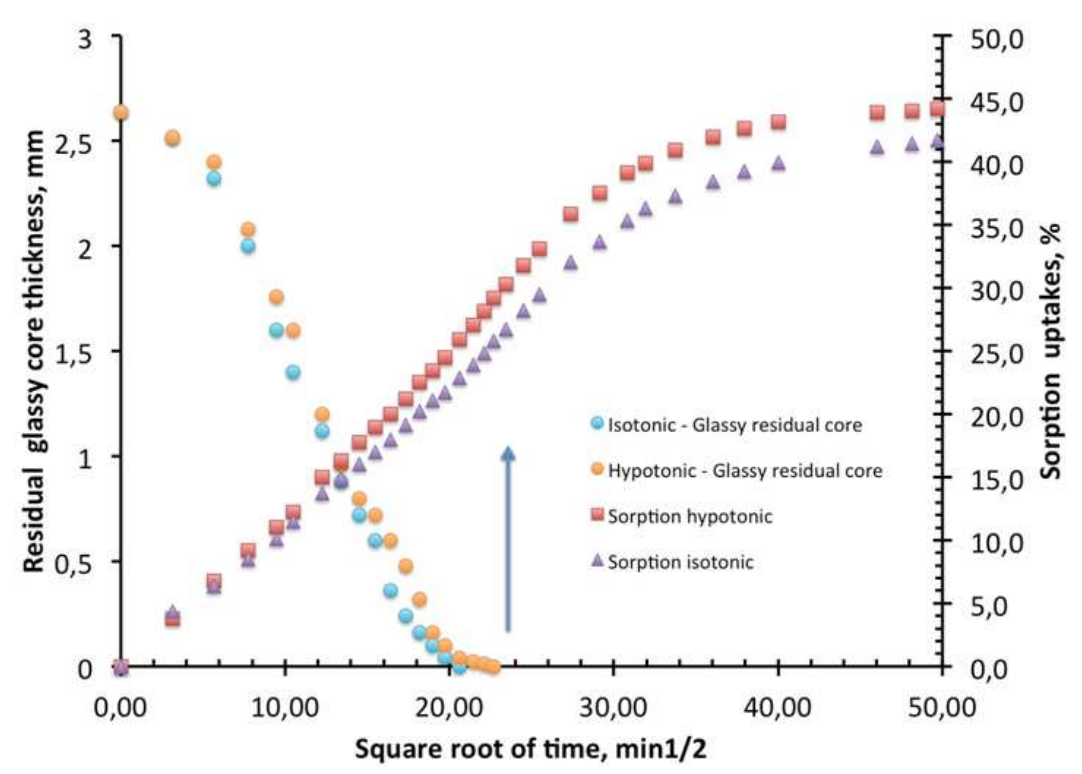

Fig. 5. Swelling and sorption kinetic as a function of the square root of the time in the $5 \%$ by volume hybrid nanocomposite in physiological $0.05 \mathrm{M}$ (hypotonic) and $0.15 \mathrm{M}$ (isotonic) $\mathrm{NaCl}$ solution

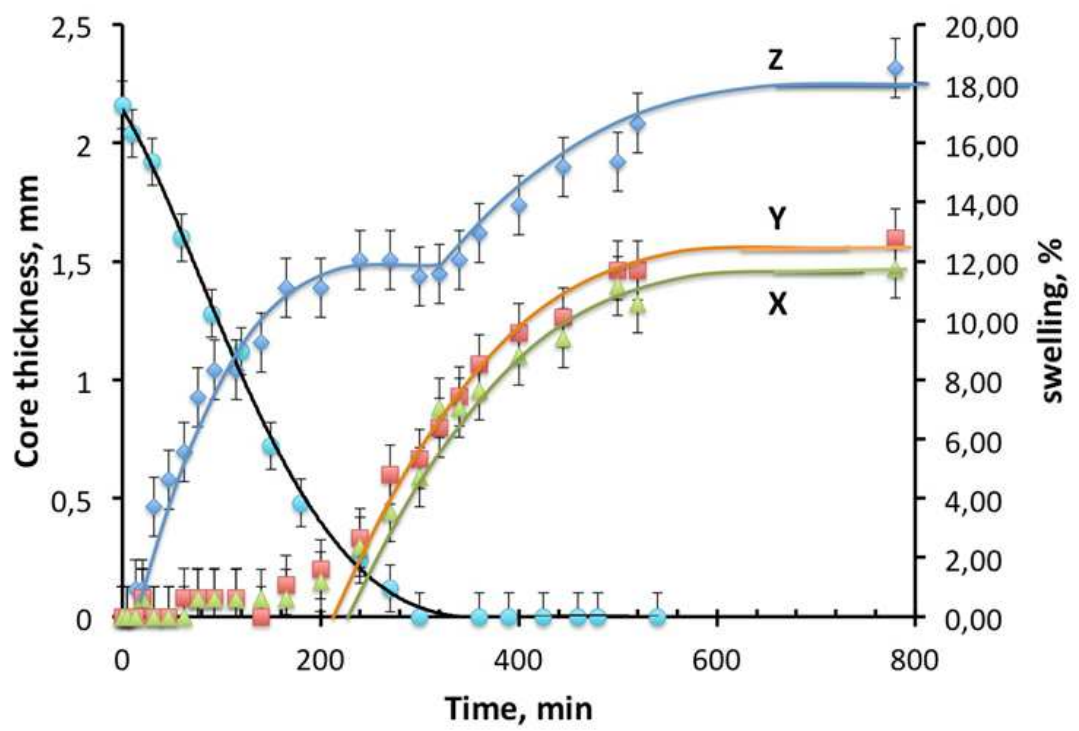

Fig. 6. Percent swelling (right axis) and glassy core thickness reduction (left axis) for the 5\% by volume fumed silica hybrid nanocomposite in physiological $0.15 \mathrm{M}$ (isotonic) $\mathrm{NaCl}$ solution

\section{Discussion}

Water plays a critical role for the physiological equilibria in the human body. Water may be eliminated from the body by loss from skin, respiration and kidney (via urine formation). These water losses have an essential role in removing toxins that permanently accumulate in the human body (intra and extra cellular). An equivalent daily volume intake can equalize the removal of water with urine. Final urine is the result of filtering of the plasmatic water (levels 180 $1 /$ day) and reabsorption ( $99 \%$ of the filtered water is reabsorbed into the renal tubule, of which approximately $66 \%$ is reabsorbed in the proximal convoluted tubule, iso-osmotic, non-adjustable; reabsorption adjustable occurs in the collector tube, cortically and medullary). Urine is only $1 \%$ of the filtered water (1-2 liters/day). Water losses (skin and respiratory) are $650-850 \mathrm{ml} /$ day.

So, about a third of water with toxins is eliminating through the skin. In addition the skin breathes like the nostrils, so it is necessary not only maintaining skin cleanliness but and its integrity. Sodium is the main extracellular cation, representing the most important 
osmotic pressure that maintains the size of the extracellular fluid volume.

Increasing the total amount of sodium in extracellular fluid is followed by an increased volume of the extracellular fluid compartment thereof and its overcharging. Increase blood pressure. Syndromes of swelling (in congestive heart failure, liver cirrhosis, nephrotic syndrome) are medical conditions with increased amount of sodium in the extracellular compartment (Abdul-Razzak et al., 2012; Ajith et al., 2009; Atasayar et al., 2009).

If the total amount of sodium in the extracellular compartment decreases, the volume decreases and it results in depletion of the extracellular compartment volume (extracellular dehydration). In this case the water supply decreases and from the blood, resulting in disorders of the entire body. In case of decrease in the volume of the sector plasma it can observed the acceleration in heart rate, decreased blood pressure in lying or standing, pale skin and cool extremities, decreased urine output, flattening the neck and forearm veins, the blood pressure occlusion. In case of loss of more than $30 \%$ of plasma volume, occurs the hypovolemic shock with cyanosis of extremities and neurological signs of cerebral hypo-perfusion (drowsiness, confusion, agitation).

Keeping the decrease of the volume of extracellular salt and water (much) longer produces an excessive consumption from the intracellular water reserves. It also produces and decreased interstitial fluid volume. Interstitial sector is partly responsible for skin elasticity and firmness. Such skin aging occurs and of the cells. With the loss of water from the cells and energy is lost. Without enough water in cells are produced less ATP molecules (even if we have enough mitochondria). Cell aging occurs and a lack of energy. Conversely, in the event of increased plasma sector, there is an increase in blood pressure.

Renin is an enzyme formed and released by the granule cells of the glomus (Ahmed et al., 2011). Renin catalyses the conversion of angiotensinogen (protein synthesized by the liver) to angiotensin I. Angiotensin I is converted by a converting enzyme into angiotensin II, which is biologically active (Covic et al., 2007).

All processes of a human body are and finely controlled by the various hormones produced by glands. In this respect it is important to know as well physiopathology of various human processes. That happens and with retention or release of sodium and water.

Such Sodium ions concentration changes can induce modification of the physical and mechanical properties of hydrophilic biomaterials if used for structural or scaffolding systems.

Our hybrid materials were designed to act as biocompatible and mechanically bioactive scaffolds for dental implant finalized to favour adaptive directionally organized $\mathrm{OB}$ growth in implanted bone. In order to achieve this result, both a proper scaffolding biocompatible and biomechanically active material has to be designed.

The biomimetic characteristic of our hybrid materials have been investigated both for mechanical and swelling properties in order to better understand all the physical and morphological modifications occurring when exposed to mutating physiological like conditions. In the case of use as scaffolding material for bone tissue engineering, physiological bone material behavior to be mimicked by the bioactive scaffolding material relates to the following aspects:

- Mechanical properties in the dry and swollen states as a function of the changing physiological fluid water contents

- Bioactivity and swelling behaviour sensitivity to external physiological modifications

Hybrid ceramo-polymeric insert swelling could stabilize implants in the bone and creates a biomechanically active interface for bone growth. Stresses on the bone can be modulated by scaffold swelling thickness choice for healthy bone growth. In vivo tests performed using these new modified oral implant confirmed the improved capability of such implants in promoting early osseointegration (Gramanzini et al., 2016).

\section{Conclusion}

All processes of a human body are and finely controlled by the various hormones produced by glands that can modify both the extracellular water content and Sodium ions concentrations:

Angiotensin II promotes salt retention through direct and indirect effects. It directly stimulates sodium reabsorption in the proximal tubule (by stimulating the exchange $\left.\mathrm{Na}^{+} / \mathrm{H}^{+}\right)$. Indirectly, it affects sodium balance by stimulating the production and release of aldosterone from the glomerular zone of the adrenal. Aldosterone induces a sodium reabsorption (and $\mathrm{K}^{+}$secretion) in the distal convoluted tubule and sewer.

Vasopressin, or antidiuretic hormone is regulated primarily by the osmolarity of body fluids, but the vasopressin increases and in volume-depleted states. This promotes water retention and restoration of body fluid volume.

Atrial natriuretic factor is a peptide synthesized by atrial myocytes and released as aresponse to atrial distension. It is effective to increase natriuresis by the action of vasodilation, the afferent arteriole with increasing DFG (glomerular filtration flow) and filtered soda task; It has direct inhibitory action on the absorption of soda in the proximal tubule and medullary 
tube collector. It has inhibitory effect on renin release, $\mathrm{ADH}$ (antidiuretic hormone) and aldosterone.

Prostaglandins have vasodilating renal action and induce the removal of sodium and water. They increase the excretion of sodium by increasing the amount of sodium filtered and all they increase water excretion by antagonizing $\mathrm{ADH}$.

The sympathetic nervous system, the reninangiotensin-aldosterone system, the natriuretic peptide atrial and the vasopressin represent the four main systems regulators who tailor their activity in response to changes in body fluid volume.

In this respect it is important to know physiopathology of various human processes that induce retention or release of sodium and water. The sympathetic nervous system, the renin-angiotensin-aldosterone system, the natriuretic peptide atrial and the vasopressin represent the four main systems regulators who tailor their activity in response to changes in body fluid volume.

Keeping the decrease of the volume of extracellular salt and water (much) longer produces an excessive consumption from the intracellular water reserves.

The volume of extracellular Sodium and water modification and decrease of interstitial fluid volume, which is partly responsible for skin elasticity and firmness, may likewise strongly influence hydrophilic biomaterial mechanical and physiological functions.

A biomimetic/physiological approach has been pursued in designing the formulation of new bioactive ceramo-polymeric hybrid materials for biomechanical stimulation and potential improved bone scaffold mineralization and ossification.

A new nanocomposites hybrid ceramo-polymeric Hydroxyl-Ethyl-Methacrylate polymer (pHEMA) filled with nanosilica particles (5\% by volume) has been chosen as optimum biomimetic material composition. This material swells (about 14\% linearly) in presence of aqueous physiological solution (when in a biological aqueous environment) picking-up to $40-45 \%$ by weight of water (depending on water contented of the external physiological medium) turning from glassy and rigid to soft and rubbery. Mechanical behavior of the proposed hybrid materials are comparable with those of bone when in the glassy state and to those of the cartilage (ligaments) when rubbery after swelling.

The use of mechanically compatible hybrid hydrogels as scaffolding materials are expected to increase prosthesis adaptation mechanisms introducing active interfaces that improve implant biomimetics while reproducing cartilage and ligaments biomechanical functions (Apicella et al., 2010; 2011; 2015; Aversa et al., 2016b; Perillo et al., 2010; Schwartz-Dabney and Dechow, 2003). Adaptive properties of bone could benefit of use of biomimetic (biomechanically compatible and bioactive) scaffold biomaterials coupled with new designed odontostomatological prostheses.

\section{Acknowledgement}

This text was acknowledged and appreciated by Assoc. Pro. Taher M. Abu-Lebdeh, North Carolina A and T State Univesity, United States, Muftah H. El-Naas PhD MCIC FICCE QAFCO Chair Professor in Chemical Process Engineering Gas Processing Center College of Engineering Qatar University, Professor Guanying Chen Harbin Institute of Technology \& SUNY Buffalo China, and (Ms.) Shweta Agarwala Senior Research Scientist at Singapore Center for 3D Printing Nanyang Technological University Singapore, whom we thanks and in this way.

\section{Author's Contributions}

All the authors contributed equally to prepare, develop and carry out this manuscript.

\section{Ethics}

This article is original and contains unpublished material. The corresponding author confirms that all of the other authors have read and approved the manuscript and no ethical issues involved.

\section{References}

Abdul-Razzak, K., K. Alzoubi, S. Abdo and W. Hananeh, 2012. High-dose vitamin C: Does it exacerbate the effect of psychosocial stress on liver? Biochemical and histological study. Experim. Toxicol. Pathol., 64: 367-371. DOI: 10.1016/j.etp.2010.09.011

Ahmed, E., H. Omar, S. Elghaffar, S. Ragb and A. Nasser, 2011. The antioxidant activity of Vitamin C, DPPD and 1-cysteine against Cisplatin-induced testicular oxidative damage in rats. Food Chem. Toxicol., 49: 1115-1121.

DOI: $10.1016 /$ j.fct.2011.02.002

Ajith, T.A., G. Abhishek, D. Roshny and N.P. Sudheesh, 2009. Co-supplementation of single and multi doses of vitamins $\mathrm{C}$ and $\mathrm{E}$ ameliorates cisplatininduced acute renal failure in mice. Experim. Toxicol. Pathol., 61: 565-571. DOI: $10.1016 /$ j.etp.2008.12.002

Apicella, A., B. Cappello, M.A. Del Nobile, M.I. La Rotonda and G. Mensitieri et al., 1993. Poly(Ethylene oxide) (PEO) and different molecular weight PEO blends monolithic devices for drug release. Biomaterials, 142: 83-90. DOI: 10.1016/0142-9612(93)90215-N

Apicella, A. and H.B. Hopfenberg, 1982. Water-swelling behavior of an ethylene-vinyl alcohol copolymer in the presence of sorbed sodium chloride. J. Applied Polymer Sci., 27: 1139-1148.

DOI: 10.1002/app.1982.070270404 
Apicella, D., R. Aversa, M. Tatullo, M. Simeone and S. Sayed et al., 2015. Direct restoration modalities of fractured central maxillary incisors: A multilevels validated finite elements analysis with in vivo strain measurements. Dental Mater., 31: e289-e305. DOI: 10.1016/j.dental.2015.09.016

Apicella, D., M. Veltri, P. Balleri, A. Apicella and M. Ferrari, 2011. Influence of abutment material on the fracture strength and failure modes of abutmentfixture assemblies when loaded in a bio-faithful simulation. Clin. Oral Implants Res., 22: 182-188. DOI: $10.1111 / \mathrm{j} .1600-0501.2010 .01979 . \mathrm{x}$

Apicella, D., R. Aversa, E. Ferro, Ianniello, D. Ianniello and A. Apicella, 2010. The importance of cortical bone orthotropicity, maximum stiffness direction and thickness on the reliability of mandible numerical models. J. Biomed. Mater. Res. Part B Applied Biomater., 93: 150-163.

DOI: $10.1002 / \mathrm{jbm} . b .31569$

Atasayar, S., H. Gürer-Orhan, B. Gürel, G. Girgin and H. Özgünes, 2009. Preventive effect of aminoguanidine compared to vitamin $\mathrm{E}$ and $\mathrm{C}$ on cisplatin-induced nephrotoxicity in rats. Experim. Toxicol. Pathol., 61: 23-32. DOI: 10.1016/j.etp.2008.04.016

Aversa, R., D. Apicella, L. Perillo, R. Sorrentino and F. Zarone et al., 2009. Non-linear elastic threedimensional finite element analysis on the effect of endocrown material rigidity on alveolar bone remodeling process. Dental Mater., 25: 678-690. DOI: 10.1016/j.dental.2008.10.015

Aversa, R., F.I.T. Petrescu, V.P. Relly and A. Apicella, 2016b. Biomimetic FEA bone modeling for customized hybrid biological prostheses development. AJAS, in press.

Aversa, R., R. Sorrentino and A. Apicella, 2016a. Biomechanically active ceramic-polymeric hybrid scaffolds for tissue engineering. Proceedings of the International Conference on Biological Sciences and Technology, (BST' 16), Atlantis Press, pp: 308-318. DOI: $10.2991 /$ bst-16.2016.46

Aversa, R. and A. Antonio, 2016. Near critical carbon dioxide sorption induced crystallization in PET. Am. J. Eng. Applied Sci. DOI: 10.3844/ofsp.10675

Babaev, V.R., L. Li, S. Shah, S. Fazio and M.F. Linton et al., 2010. Combined vitamin $\mathrm{C}$ and vitamin E deficiency worsens early atherosclerosis in apolipoprotein E-Deficient mice. Arteriosclerosis, Thrombosis Vascular Biol., 30: 1751-1757. DOI: 10.1161/ATVBAHA.110.209502

Buzea, E., F.1. Petrescu, L. Nanut, C. Nan and M. Neacsa, 2015. Mechatronic system to determine the concentration of carotenoids, analele Univers. Craiova Biologie Horticultura Tehn. Prel. Prod. Agr. Ing. Med., 20: 371-376.

Comerun, H.U., 1986. Six-year results with a microporous-coated metal hip prosthesis, Clin. Orthop., 208: 81-83. PMID: 3522027
Covic, M., A. Covic, P.G. Tatomir and L. Segall, 2007. Manual de Nefrologie. 1st Edn., Polirom Publisher, pp: 448.

Davis, P.A., S.J. Huang, L. Nicolais and L. Ambrosio, 1991. Modified PHEMA Hydrogels. In: High Performance Biomaterials: A Complete Guide to Medical and Pharmceutical Applications. Szycher, M., (Ed.), CRC Press, ISBN-10: 0877627754, pp: 343-368.

Filmon, R., F. Grizon, M.F. Baslie and D. Chappard, 2002. Effects of negatively charged groups (carboxymethyl) on the calcification of poly(2hydroxyethyl methacrylate). Biomaterials, 23: 3053-3059. DOI: 10.1016/S0142-9612(02)00069-8

Frost, H.M., 2004. A 2003 update of bone physiology and Wolff's law for clinicians. Angle. Orthod., 74: 3-15. PMID: 15038485

Frost, H.M., 1964. Mathematical Elements of Lamellar Bone Remodelling. 1st Edn., Thomas, Springfield, pp: 127.

Frost, H.M., 1990. Skeletal structural adaptations to mechanical usage (SATMU): 2. Redefining Wolff's law: The remodeling problem. Anat. Rec., 226: 414-422. PMID: 2184696

Frost, H.M., 1994. Wolff's law and bone's structural adaptations to mechanical usage: An overview for clinicians. Angle Orthod., 64: 175-188.

PMID: 8060014

Gramanzini, M., S. Gargiulo, F. Zarone, R. Megna and A. Apicella et al., 2016. Combined microcomputed tomography, biomechanical and histomorphometric analysis of the peri-implant bone: A pilot study in minipig model. Dental Mater., 32: 794-806. DOI: 10.1016/j.dental.2016.03.025

Halpin, J.C. and J.L. Kardos, 1976. The Halpin-Tsai equations: A review. Polymer Eng. Sci., 16: 344-352. DOI: $10.1002 /$ pen.760160512

Holley, R.H., H.B. Hopfenberg and V. Stannett, 1970. Anomalous transport of hydrocarbons in polystyrene. Polymer Eng. Sci., 10: 376-382. DOI: 10.1002/pen.760100612

Kabra, B., S.H. Gehrke, S.T. Hwang and W. Ritschel, 1991. Modification of the dynamic swelling behavior of poly(2-hydroxyethyl methacrylate) in water. J. Applied Polym. Sci., 42: 2409-2416. DOI: 10.1002/app.1991.070420906

Montheard, J.P., M. Chatzopoulos and D. Chappard, 1992. 2-Hydroxyethyl Methacrylate (HEMA): Chemical properties and applications in biomedical fields. J. Macromol. Sci. Macromol. Rev., 32: 1-34. DOI: $10.1080 / 15321799208018377$

Nicolais, L., A. Apicella and C. de Notaristefano, 1984. Time-temperature superposition of $n$-hexane sorption in polystyrene. J. Membrane Sci., 18: 187-196. DOI: $10.1016 / \mathrm{S} 0376-7388(00) 85033-4$ 
Peluso, G., O. Petillo, J.M. Anderson, M. Ambrosio and L. Nicolais et al., 1997. The differential effects of poly(2-hydroxyethyl methacrylate) and poly(2hydroxyethyl methacrylate)/poly(caprolactone) polymers on cell proliferation and collagen synthesis by human lung fibroblasts. J. Biomed. Mater. Res., 34: $\quad 327-336$. DOI: $10.1002 /(\mathrm{SICI}) 1097-$ 4636(19970305)34:3<327::AID-JBM7>3.0.CO;2-M

Perillo, L., R. Sorrentino, D. Apicella, A. Quaranta and E. Gherlone et al., 2010. Nonlinear visco-elastic finite element analysis of porcelain veneers: A submodelling approach to strain and stress distributions in adhesive and resin cement. J. Adhes. Dent., 12: 403-413. PMID: 20157681

Petrescu, F.1., E. Buzea, L. Nănuţ, M. Neacşa and C. Nan, 2015. The role of antioxidants in slowing aging of skin in a human, Analele Univers. Craiova Biologie Horticultura Tehn. Prel. Prod. Agr. Ing. Med., 20: 567-574.

Schiraldi, C., A. D' Agostino, A. Oliva, F. Flamma and A. De Rosa et al., 2004. Development of hybrid materials based on hydroxyethylmethacrylate as supports for improving cell adhesion and proliferation. Biomaterials, 25: 3645-3653.

DOI: $10.1016 /$ j.biomaterials.2003.10.059
Schwartz-Dabney, C.L. and P.C. Dechow, 2003. Variations in cortical material properties throughout the human dentate mandible. Am. J. Phys. Anthropol., 120: 252-277. DOI: 10.1002/ajpa.10121

Sorrentino, R., R. Aversa, V. Ferro, T. Auriemma and F. Zarone et al., 2007. Three-dimensional finite element analysis of strain and stress distributions in endodontically treated maxillary central incisors restored with diferent post, core and crown materials. Dent Mater., 23: 983-993.

DOI: $10.1016 /$ j.dental.2006.08.006

Sorrentino, R., D. Apicella, C. Riccio, E.D. Gherlone and F. Zarone et al., 2009. Nonlinear visco-elastic finite element analysis of different porcelain veneers configuration. J. Biomed. Mater. Res.-Part B Applied Biomater., 91: 727-736.

DOI: $10.1002 /$ jbm.b.31449

Toyrasa, J., T. Lyyra-Laitinena, M. Niinimäkib, R. Lindgrenc and M.T. Nieminenb et al., 2001. Estimation of the Young's modulus of articular cartilage using an arthroscopic indentation instrument and ultrasonic measurement of tissue thickness. J. Biomechan., 34: 251-256. DOI: 10.1016/S0021-9290(00)00189-5

Wolff, J., 1892. Das Gesetz der Transformation der Knochen. A. Hirschwald. 